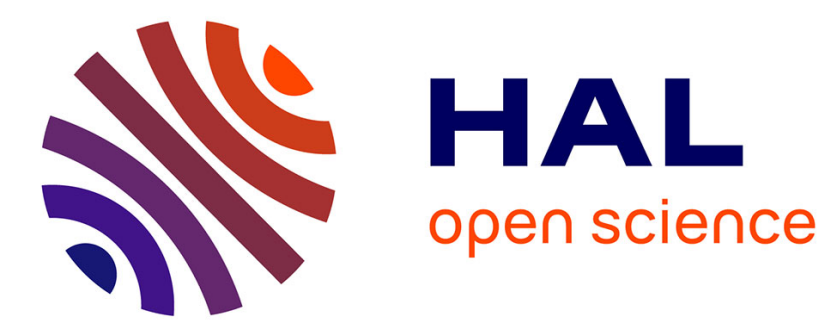

\title{
Discrete flatness for non linear systems FDI
}

\author{
Nan Zhang, Andrei Doncescu, Felix Mora-Camino
}

\section{To cite this version:}

Nan Zhang, Andrei Doncescu, Felix Mora-Camino. Discrete flatness for non linear systems FDI. BWCCA 2010, 5th International Conference on Broadband, Wireless Computing, Communication and Applications, Nov 2010, Fukuoka, Japan. 10.1109/BWCCA.2010.119 . hal-01021489

\section{HAL Id: hal-01021489 \\ https://hal-enac.archives-ouvertes.fr/hal-01021489}

Submitted on 31 Oct 2014

HAL is a multi-disciplinary open access archive for the deposit and dissemination of scientific research documents, whether they are published or not. The documents may come from teaching and research institutions in France or abroad, or from public or private research centers.
L'archive ouverte pluridisciplinaire HAL, est destinée au dépôt et à la diffusion de documents scientifiques de niveau recherche, publiés ou non, émanant des établissements d'enseignement et de recherche français ou étrangers, des laboratoires publics ou privés. 


\section{Discrete Flatness for Non Linear Systems FDI}

\author{
Nan Zhang and Andrei Doncescu \\ LAAS CNRS \\ LARA-ENAC \\ Toulouse, France \\ e-mail: andrei.doncescu@laas.fr
}

\author{
Felix Mora-Camino \\ LAAS CNRS \\ LARA-ENAC \\ Toulouse, France \\ e-mail: felix.mora@enac.fr
}

\begin{abstract}
This Fault detection is essential for the survivability of many systems. Since many systems present highly nonlinear dynamics, the applicability of general fault detection techniques designed mainly for linear systems is very questionable. The purpose of this communication is to investigate the usefulness of the differential flatness theory of a non linear system such as a four rotor aircraft to design an efficient fault detection scheme. In this communication, after introducing the concept of difference flat nonlinear systems, a fault detection scheme based on difference flatness.
\end{abstract}

Keywords-component; Differential flatness; Fault detection; Discrete system; Rotorcraft flight mechanics;

\section{INTRODUCTION}

In the last decade a large amount of interest has raised for new fault detection and identification (FDI) approaches for non linear systems. However, few results have been obtained through purely non linear approaches. Differential flatness, a property of some nonlinear dynamic systems, introduced by Fliess et al. from the theory of differential geometry, has made possible the development of new tools to control effectively nonlinear systems. Many dynamic non linear systems have been proved to be differentially flat and the differential flatness of conventional and non conventional aircraft dynamics has been proven in different situations. While there are many different approaches to cope with fault detection in the case of linear systems, this is not the case with non linear systems and in this paper we introduce a fault detection technique applicable to difference flat non linear systems. In the first part of this paper, the main concepts relative to difference flatness applied to discrete dynamical systems are particularly considered. Then a new approach, based on the redundancy between flat outputs and direct state component measurements, is proposed. To take into account measurement errors as well as modeling errors to perform fault detection tests in this non linear context, probabilistic distributions are generated on-line. Finally, the application of the proposed approach is to a rotorcraft subject to faults characterized by parameter shifts is discussed.

\section{DIFFERENCE FLAT SYSTEMS}

Consider a non-linear system whose discrete time dynamics are given from an initial state $\underline{X}_{0}$ by :

$$
\underline{X}_{k+1}=\underline{X}_{k}+f\left(\underline{X}_{k}, \underline{U}_{k}\right)
$$

for $k \in N$, where $\underline{X}_{k} \in R^{n}, \underline{U}_{k} \in R^{m}, f$ is a smooth vector field of $\underline{X}_{k}$ and $\underline{U}_{k}$ which are respectively the state and the input vectors of this system at time $\mathrm{k}$. It is supposed here that each input has an independent effect on the state dynamics:

$$
\partial f / \partial u_{i} \neq \partial f / \partial u_{j}, \quad i \neq j, \quad \text { with } \quad i, j \in\{1, \ldots, m\}
$$

\section{A. Difference Flatness of order $(p, q)$}

The system given by (1) is said to be difference flat of order $(p, q)$, where $p$ and $q$ are integers, if there exists a measurable output $\underline{Y} \in R^{m}$ :

$$
\underline{Y}_{k}=h\left(\underline{X}_{k}\right)
$$

where $\mathrm{h}$ is a smooth vector field of $\underline{X}_{k}$, such as it is possible to write:

$$
\begin{aligned}
\underline{X}_{k} & =\eta\left(\underline{Y}_{k+p}, \underline{Y}_{k+p-1}, \ldots, \underline{Y}_{k-q}\right) \\
\underline{U}_{k} & =\xi\left(\underline{Y}_{k+p+1}, \underline{Y}_{k+p}, \ldots, \underline{Y}_{k-q}\right)
\end{aligned}
$$

where $\eta($.$) is a function of \underline{Y}_{j}$ and its values from $k+p_{j}$ back to order $k-q_{j}$, and that $\xi($.$) is a function of$ $\underline{Y}_{j}$ and its values from $k+p_{j}+1$ back to $k-q_{j}$, for $\mathrm{j}=1$ to $\mathrm{m}$. Here $\mathrm{p}$ and $\mathrm{q}$ are given by:

$$
p=\max \left\{p_{j}\right\} \text { and } q=\max \left\{q_{j}\right\} \text {, where } \mathrm{j}=1 \text { to } \mathrm{m}
$$

For $\mathrm{j}=1$ to $\mathrm{m}, p_{j}$ is called the discrete relative degree of output $\underline{Y}_{j}$, while $p_{j}+q_{j}$ is the time span of the dynamics of output $\mathrm{j}$. It is easy to show that:

$$
\sum_{j=1}^{m}\left(p_{j}+q_{j}\right) \leq n
$$


For example, if we consider the following discrete dynamics:

$$
\left\{\begin{array}{c}
x_{1, k+1}=x_{2, k}^{2}+\left(u_{k}\right)^{2} \\
x_{2, k+1}=x_{2, k} /\left(1+x_{3, k}\right) \\
x_{3, k+1}=x_{3, k} \cdot x_{4, k} \\
x_{4, k+1}=u_{k}
\end{array}\right.
$$

It is easy to show that $\mathrm{y}_{\mathrm{k}}=\mathrm{x}_{2, \mathrm{k}}$ is a flat output for this system with an order $(2,1)$. Indeed it is possible to write:

$$
\left\{\begin{array}{c}
x_{1, k}=y_{k-1}+\left(y_{k+1} \cdot\left(y_{k}-y_{k+2}\right) / y_{k+2} \cdot\left(y_{k}-y_{k+1}\right)\right)^{2} \\
x_{2, k}=y_{k} \\
x_{3, k}=y_{k} / y_{k+1}-1 \\
x_{4, k}=y_{k+1} \cdot\left(y_{k+1}-y_{k+2}\right) / y_{k+2} \cdot\left(y_{k}-y_{k+1}\right)
\end{array}\right.
$$

where

$$
u_{k}=y_{k+2} \cdot\left(y_{k+2}-y_{k+3}\right) / y_{k+3} \cdot\left(y_{k+1}-y_{k+2}\right)
$$

\section{B. 2.2 Nominal State Reconstruction}

It appears in the case of difference flat systems that perfect on-line state reconstruction is possible in theory through two steps:

At current time, the set of measurements $\left(\underline{Y}_{k-q}, \underline{Y}_{k-q+1}, \cdots, \underline{Y}_{k+p-1}, \underline{Y}_{k+p}\right)$ is available, so it is possible when the model of the discrete dynamics and the measurements are assumed to be perfect, to compute the exact value of the state of the system at time $\mathrm{k}$ by the discrete flat relation:

$$
\underline{\tilde{X}}_{k}=\eta\left(\underline{Y}_{k+p_{1}}, \ldots, \underline{Y}_{k-q_{1}}\right)
$$

Then, starting from this value and using repeatedly the discrete state equation (1) from time $\mathrm{h}=\mathrm{k}$ to time $\mathrm{k}+\mathrm{p}-1$ with the past known inputs $U_{h}$ :

$$
\underline{\tilde{X}}_{h+1}=\underline{\tilde{X}}_{h}+f\left(\underline{\tilde{X}}_{h}, \underline{U}_{h}\right)
$$

we get the current state value $\underline{\widetilde{X}}_{h+p}$.

For example, in the case of discrete dynamical system (8), in order (2.1), the state of the system at time $\mathrm{k}$ can be calculated by:

$$
\left\{\begin{array}{lr}
\widetilde{x}_{1, k}=y_{k-1}+\left(y_{k+1} \cdot\left(y_{k}-y_{k+2}\right) / y_{k+2} \cdot\left(y_{k}-y_{k+1}\right)\right)^{2} & \widetilde{x}_{2, k}=y_{k} \\
\tilde{x}_{3, k}=y_{k} / y_{k+1}-1 & \widetilde{x}_{4, k}=y_{k+1} \cdot\left(y_{k+1}-y_{k+2}\right) / y_{k+2} \cdot\left(y_{k}-y_{k+1}\right)
\end{array}\right.
$$

then at a time $\mathrm{k}$ we have:

$$
\left\{\begin{array}{cc}
\tilde{x}_{1, k+1}=\tilde{x}_{2, k}^{2}+\left(u_{k}\right)^{2} & \tilde{x}_{2, k+1}=\tilde{x}_{2, k} /\left(1+\tilde{x}_{3, k}\right) \\
\widetilde{x}_{3, k+1}=\widetilde{x}_{3, k} \cdot x_{4, k} & \tilde{x}_{4, k+1}=u_{k}
\end{array}\right.
$$

Finally, at time $\mathrm{k}+2$ :

$$
\left\{\begin{array}{cc}
\widetilde{x}_{1, k+2}=\widetilde{x}_{2, k+1}^{2}+\left(u_{k+1}\right)^{2} & \widetilde{x}_{2, k+2}=\widetilde{x}_{2, k+1} /\left(1+\widetilde{x}_{3, k+1}\right) \\
\widetilde{x}_{3, k+2}=\widetilde{x}_{3, k+1} \cdot x_{4, k+1} & \widetilde{x}_{4, k+2}=u_{k+1}
\end{array}\right.
$$

Unfortunately, discrete models and actual measurements present in general systematic errors and fault detection above scheme will lead to detection errors (false breakdown, problems not detected) and cannot be used directly.

\section{FAULT DETECTION FOR DIFFERENCE FLAT SYSTEMES}

The proposed detection scheme is based on the redundancy of information which is present when considering simultaneously flat outputs and some state components of a system subject to faults. So, here we consider that an output composed of a flat output vector and $\mathrm{p}$ additional components of the state vector is available at each time period:

$$
\underline{Z}_{k}=\left[\begin{array}{c}
\underline{Y}_{k}^{\mu} \\
x_{i_{1}}^{\mu} \\
\vdots \\
x_{i_{p}}^{\mu}
\end{array}\right]=\left[\begin{array}{c}
\underline{Y}_{k} \\
x_{i_{1}} \\
\vdots \\
x_{i_{p}}
\end{array}\right]+\left[\begin{array}{l}
\underline{v}_{k} \\
\underline{v}_{k}
\end{array}\right]
$$

with $m+p \leq n$ where $\underline{v}_{k} \in R^{m}$ and $\underline{v}_{k} \in R^{p}$ are measurements errors.

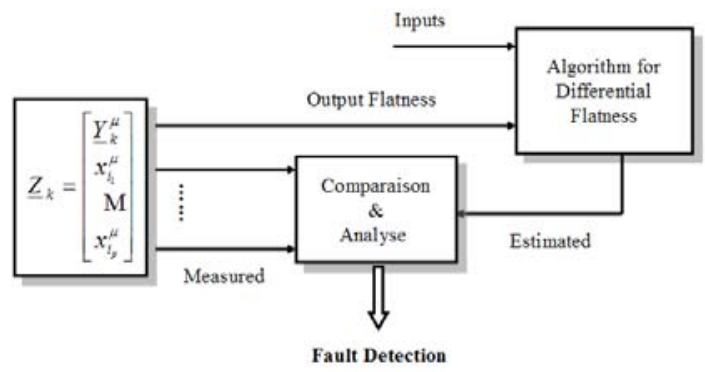

Figure 1 : Generation of Fault detection by Differential Flatness

Since in theory it is possible to reconstruct the state of the system from past and present flat outputs and inputs, at current time $\mathrm{k}+\mathrm{p}$ it will be possible to compute residuals such as:

$$
\delta X_{j, k+p}=X_{i_{j}}^{\mu}-\tilde{X}_{i_{j}} \quad \mathrm{j}=1 \text { à } \mathrm{p}
$$

and considering the accuracy of the measurement channels and of the discredited model, it should be possible to set thresholds $\sigma_{j}^{X}, j=1, \cdots, p$ to detect faults in the system. Then the satisfaction of tests such as:

$$
\text { if } \quad \exists j \in\{1, \cdots, p\}:\left|\delta X_{j, k+p}\right|>\sigma_{j}^{X}
$$

will indicate the presence of a fault with some probability $\pi_{j}, j \in\{1, \cdots, p\}$. 
It is clear that the effectiveness of this method of fault detection is directly dependent on the choice of levels of these thresholds. To investigate this point, additional assumptions are introduced:

It is assumed that the measurement errors $\underline{v}_{k}$ follow stochastic processes like Gaussian white noise with zero mean. This means that for the vector $\underline{v}_{k}$ we have:

$$
E\left\{\underline{v}_{k}\right\}=\underline{0}
$$

and with constant variances given by:

where

$$
E\left\{\underline{v}_{k} \underline{v}_{h}{ }^{t}\right\}=\operatorname{diag}\left\{V_{i}^{2}, i=1 \text { to } m\right\} \delta_{k, h}
$$

$$
\delta_{k, h}=0 \text { if } k \neq h \text { and } \delta_{k, k}=1
$$

In the same way, we suppose that:

and

$$
E\left\{v_{k}\right\}=\underline{0}
$$

where

$$
E\left\{\underline{v}_{k} \underline{v}_{h}{ }^{t}\right\}=\operatorname{diag}\left\{\Delta_{i}{ }^{2}, i=1 \text { to } p\right\} \delta_{k, h}
$$

$$
\delta_{k, h}=0 \text { if } k \neq h \text { and } \delta_{k, k}=1
$$

The modeling error can be also approximated by additive Gaussian white noises such that the state dynamics of the system under consideration can be rewritten:

$$
\underline{X}_{k+1}=\underline{X}_{k}+f\left(\underline{X}_{k}, \underline{U}_{k}\right)+\underline{\omega}_{k}
$$

where $\underline{\omega_{k}}$ is a Gaussian white noise vector of dimension $\mathrm{n}$ such as:

$$
E\left\{\underline{\omega}_{k}\right\}=\underline{0}
$$

and

$$
E\left\{\underline{\omega}_{k} \underline{\omega}_{h}{ }^{t}\right\}=\operatorname{diag}\left\{W_{i}^{2}, i=1 \text { to } n\right\} \delta_{k, h}
$$

To define the appropriate probability levels used in the fault detection test, see relation (18), since the flatness relation (11) and the state equation (12) are in general non-linear. Then, it is necessary to generate on line the probability distribution of the error of the current state estimations.

\section{GENERATION OF STATE ESTIMATES DISTRIBUTION}

It is possible to generate through different realizations of the modeling and measurement errors, statistics for the estimates at current time $\mathrm{k}+\mathrm{p}$ of the state of the difference flat system. Here we will limit us to first and second order statistics.

We get first estimates at time $\mathrm{k}, 0 \underline{X}_{k}^{\left(i^{p}, \cdots, i^{-q}\right)}$, where the vectors of indexes $i^{h}$ are such as:

Then we get:

$$
\underline{i}^{h} \in N^{m}, h \in\{p, p-1, \cdots,-q\}
$$

$$
\underline{X}_{k-h}^{\left(\underline{i}^{p}, \cdots, \underline{i}^{-q}\right)}=\eta\left(\underline{Y}_{k+p}^{i^{p}}, \cdots, \underline{Y}_{k-q}^{i^{-q}}\right)
$$

For each choice s of $\underline{i}^{h}, i_{j}^{h, s}$ the flat output component $\mathrm{j}=1$ to $\mathrm{m}$, present in $\eta$ is computed according to:

$Y_{j, h}^{i_{j}^{h}}=Y_{j, k+h}^{\mu}+i_{j}^{h, s} \cdot V_{j} \quad i_{j}^{h, s} \in\{-N, \cdots, N\}, \quad h=p, p-1, \cdots,-q$

Let $\rho_{j, h}^{i_{j}^{h, s}}$ be the associated probability given by:

$$
\rho_{j, h}^{i_{j}^{h, s}}=\frac{-\exp \left(i_{j}^{h}\right)^{2}}{\sqrt{2 \pi} \cdot V_{j}} \quad i=-N \text { to } N
$$

Let $r_{\max }$ be the maximum number of different estimates which is generated according to relations (29) and (30) at time $\mathrm{k}+\mathrm{p}$ for each component of the state of the difference flat system at time $\mathrm{k}$. The number of $r_{\max }$ is such as:

$$
r_{\max } \leq(2 N+1)^{m \cdot(p+q+1)}
$$

Since this number can be excessive (for $\mathrm{N}=5, \mathrm{~m}=3, \mathrm{p}=2$, $\mathrm{q}$ $=1$ we get $r_{\max } \approx 3.10^{12}$ ), the number of choices for $\mathrm{s}$ must be strongly limited. For a single choice of $\mathrm{s}$ among $\{-N, \cdots, N\}$, we generate for $\mathrm{N}=5, \mathrm{~m}=3, \mathrm{p}=2$ and $\mathrm{q}=1, \mathrm{r}_{\max }=12$ different values for each state component and then $r_{\max }=4090$ different values when two different choices are done for $\mathrm{s}$.

By similarity with the particular filtering approach, we will call particle each generated state $\underline{X}_{k}^{\left(i^{p}, \cdots, i^{-q}\right)}$ for time $\mathrm{k}$ from measurements $\underline{Y}_{k-q}^{\mu}$ to $\underline{Y}_{k+p}^{\mu}$. Let $\underline{\hat{X}}_{k}^{r}$ be the rth estimate of the state for period $\mathrm{k}$ generated at period $\mathrm{k}+\mathrm{p}, \mathrm{r}=1$ to $r_{\max }$.

For each particle it has until the current time:

with

$$
\underline{\breve{X}}_{h+1}^{\left(r_{k}, \cdots, r_{h}, r_{h+1}\right)}=\underline{X}_{h}^{\left(r_{k}, \cdots, r_{h}\right)}+f\left(\underline{X}_{h}^{\left(r_{h}, \cdots, r_{h}\right)}, \underline{U}_{h}, \underline{\breve{C}}_{h}^{r_{h}}\right)
$$

$$
r_{\max }^{h+1}=2 \cdot r_{\max }^{h}
$$

For $r_{h}=1$ to $r_{\max }^{h}$ initial conditions will be set by:

$$
\underline{X}_{k}^{\left(r_{k}\right)}=\underline{\hat{X}}_{k}^{r} \quad \text { with } \quad r=1 \text { to } r_{\max }^{k} \quad \text { and } \quad r_{\max }^{k}=r_{\max }
$$

where $\underline{\omega}_{h}^{r_{h}}$ is a random try for the Gaussian vector $\underline{\omega}_{h}$ to which is associated the probability:

$$
\alpha_{h}^{r}=\prod_{s=1}^{n}\left(\frac{1}{\sqrt{2 \pi} \cdot W_{s}} \cdot \exp -\left(\breve{\omega}_{s, h}^{r} / W_{s}\right)^{2} / 2\right)
$$

Then, with the chosen generation process in (30) (31), we get $2^{p} r_{\max }$ different estimates of the current state of difference flat system. Each of this estimates are characterized by the vectors of indexes such as: 
(36)

$$
\left(r_{k}, r_{k+1}, \cdots ; r_{k+p}\right) \quad \text { with } \quad r_{h} \in\left\{1, \cdots ; 2^{h-k} r_{\max }\right\}
$$

Let the weights $P_{j k+p}^{\left(r_{k}, \cdots, r_{k+p}\right)}$ be given by:

$$
P_{j k+p}^{\left(r_{,}, \cdots, r_{k+p}\right)}=\left(\prod_{h=k-q}^{k+p} \rho_{j, h}^{i_{h}, s} \cdot \alpha_{h}^{r_{h}}\right) / \sum_{r_{k-q}} \cdots \sum_{r_{k+p}}\left(\prod_{h=k-q}^{k+p} \rho_{j, h}^{i_{h}, s} \cdot \alpha_{h}^{r_{h}}\right)
$$

The generation process for state distribution at period $k+p$ if composed of two stages:

- The random generation of the state distribution at the period $\mathrm{k}$ through flat differential equation.

- The random generation of state distribution at period $\mathrm{k}+\mathrm{p}$ through state equation propagation from period $\mathrm{k}$ to period $\mathrm{k}+\mathrm{p}$.

Then an estimate of the mean value of $\underline{X}_{k+p}$ is given by:

$$
\underline{\bar{X}}_{k+p}=\sum_{r_{k-q}} \cdots \sum_{r_{k+p}}\left(P_{k+p}^{\left(r_{k}, \cdots, r_{k+p}\right)} \underline{X}_{k+p}^{\left(r_{k}, \cdots, r_{k+p}\right)}\right)
$$

with an estimate of the standard deviation of $\widetilde{X}_{j k+p}$ given by:

$$
\widetilde{V}_{j k+p}=\sum_{r_{k-q}} \cdots \sum_{r_{k+p}}\left(P_{k+p}^{\left(r_{k}, \cdots, r_{k+p}\right)}\left(X_{j_{k+p}}^{\left(r_{k}, \cdots, r_{k+p}\right)}-\bar{X}_{j k+p}\right)^{2}\right.
$$

It is now possible to make a comparison between the probabilistic noisy measured values and estimated values.

\section{TEST OF FAULT DETECTION}

In the preceding paragraph has generated a cloud of weighted estimates for the components of state vector. With the statistics of order 1 and 2 have been calculated, it is possible to define a Gaussian distribution approximating $\tilde{X}_{j k+p}$ that of by:

$$
\widetilde{f}_{j k+p}(x)=\frac{1}{\sqrt{2 \pi \widetilde{V}_{j k+p}}} \exp \left(-\left(x-\bar{X}_{j k+p}\right)^{2} /\left(2 \widetilde{V}_{j k+p}\right)\right)
$$

For the measures we can adopt the following probability distribution follows the Gaussian model around the actual measurement:

$$
f_{j k+p}(x)=\frac{1}{\sqrt{2 \pi \Delta_{j}}} \exp \left(-\left(x-X_{j k+p}^{\mu}\right)^{2} /\left(2 \Delta_{j}\right)\right)
$$

Then, the fault detection based on a difference between measurement and estimation of $X_{j, k+p}$, can be achieved by comparing the distributions given by the relations (40) and (41). The Figure 2 illustrates a comparison of this and its difficulties.

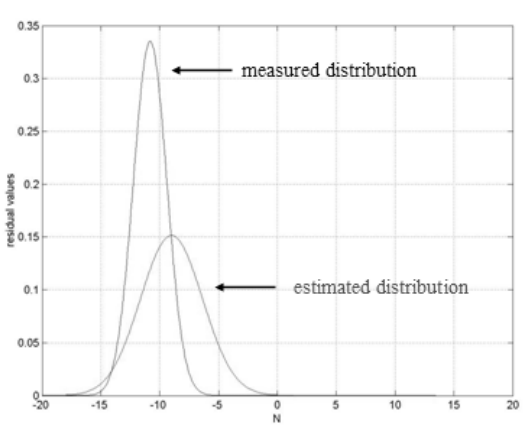

Figure 2. Comparison of measurement and estimation

We can establish probabilities of threshold, for example:

If

$$
\bar{X}_{j k+p} \leq X_{j k+p}^{\mu}:
$$

then

$$
\begin{aligned}
P\left(\left|\widetilde{X}_{j k+p}-X_{j k+p}\right| \geq \sigma_{j}^{X}\right)=\int_{-\infty}^{+\infty} \tilde{f}_{j k+p}(x)\left(\int_{-\infty}^{x+\sigma \sigma_{j}^{X}} f_{j k+p}(y) d y\right) d x \\
\bar{X}_{j k+p} \geq X_{j k+p}^{\mu}:
\end{aligned}
$$

If

then

$$
P\left(\left|\tilde{X}_{j k+p}-X_{j k+p}\right| \geq \sigma_{j}^{X}\right)=\int_{-\infty}^{+\infty} f_{j k+p}(x)\left(\int_{-\infty}^{x+\sigma_{j}^{X}} \widetilde{f}_{j k+p}(y) d y\right) d x
$$

A fault is considered detected when the following condition is satisfied:

$$
\min _{j \in\{1, \cdots, p\}} P\left(\left|\tilde{X}_{j k+p}-X_{j k+p}\right| \geq \sigma_{j}^{X}\right)>\pi_{\text {min }}
$$

where $\pi_{\min }$ is a minimum threshold for the declaration of the fault.

\section{FAULT IDENTIFICATION}

So far we have introduced and developed a new method capable of fault detection in a nonlinear dynamic system. Then we try to diagnose a fault by proposing a method that is capable of identifying faults detected.

How it was set initially locate a failure is action that is to identify at what level the fault may prevent proper operation of the process. Here below a new method is proposed. This method is successful in localization a fault by analyzing the information that we obtain the model of nonlinear dynamic system flatness. Through differential flatness was able to determine an estimate of some components of state vector compared with direct measurements of it. This will be the analysis of the evolution of the vector of errors corresponding to localize the fault.

Initially the matrix equivalence is a binary matrix consisting of column vectors given by:

$$
\underline{Z}_{k} \rightarrow \underline{A}_{k}=\left[\begin{array}{c}
\delta_{1, k} \\
\vdots \\
\delta_{p, k}
\end{array}\right]
$$


where $\delta_{i, k}$ belongs to[0,1]. The first rules $\underline{A}_{k}$ that allow writing the vector syndrome are:

$$
\begin{gathered}
\delta_{i, k}=0 \text { if }\left|x_{i, k}^{\mu}-\hat{x}_{i, k}\right|<\sigma_{i, k} \\
\delta_{i, k}=f\left(\sigma_{i, k}\right) \text { if }\left|x_{i, k}^{\mu}-\hat{x}_{i, k}\right|>\sigma_{i, k}
\end{gathered}
$$

where $x_{i, k}^{\mu}$ indicate the measured value and $\hat{x}_{i, k}$ is the estimated value. What you get is a vector corresponding to a kind of snapshot of the operating condition of the state variables at time $k$, where the presence of a value indicates that the variable is out of nominal conditions and therefore corresponds to a fault. Otherwise, a vector so all values are equal to zero would represent a system that works properly.

Considering a periods of time $t_{k}$ to $t_{k-K}$ where $K$ is the depth of the matrix $M_{k}$ equivalence, we obtain:

$$
M_{k}=\left[\begin{array}{lllll}
\underline{A}_{k-K} & \underline{A}_{k-K+1} & \cdots & \underline{A}_{k-1} & \underline{A}_{k}
\end{array}\right]
$$

So before the time when $t_{k 0}$ is a fault, the matrix of equivalence is given by:

$$
M_{k}=\left[\begin{array}{lllll}
\underline{0} & \underline{0} & \cdots & \underline{0} & \underline{0}
\end{array}\right] \in R^{p} \times R^{K}
$$

At time $t_{k 0}$, we have :

$$
M_{k}=\left[\begin{array}{lllll}
\underline{0} & \underline{0} & \cdots & \underline{0} & \underline{A}_{k 0}
\end{array}\right] \text { with } \underline{A}_{k 0} \neq \underline{0}
$$

so, we can write:

$$
M_{k}=\left[m_{j h}^{k}\right]
$$

where $m_{j h}^{k}$ is $j^{\text {th }}$ component of $h^{\text {th }}$ column vector of matrix equivalence $\mathrm{M}_{\mathrm{k}}$.

The propagation time $\tau$ of the fault supposedly less than K, is defined by the period of time from which the vector Ak continually changing

$$
\tau=\min _{k>0} \text { such as } \underline{A}_{k}=\underline{A}_{k-1}
$$

Thus, it is assumed here that the presence of a fault is manifested by propagation in the system and therefore transformation vectors syndromes $A_{h}$.

Assume that it is possible to characterize a fault $i$ by its nominal propagation $\pi \mathrm{i}$ given by:

$$
\pi_{i}=\left\{\tau_{i}, P^{\tau}=\left[p_{j h}\right]=\left[\underline{P}_{\tau_{i}}^{i}, \underline{P}_{\tau_{i}-1}^{i}, \cdots, \underline{P}_{0}^{i}\right]\right\} \quad i \in I
$$

where I is a set of faults identified.

The identification of the fault can be done for example by look for the minimum of:

$$
\min _{i \in||_{z}=\tau} \sum_{h=1}^{\tau+1} \sum_{j=1}^{p}\left(m_{j h}^{k}-p_{j h}\right)^{2}=\mathrm{D}^{*} \text { obtained } \mathrm{i} \in \mathrm{I}(0)^{*}
$$

we assume that the obtained $i \in \mathrm{I}(0)^{*}$. Indeed, it is assumed that there may be several equally plausible explanations $\left(\left|\mathrm{I}(0)^{*}\right|>1\right)$.

If $\mathrm{D}^{*}=0$, considering that $\mathrm{I}(0)^{*}=\left\{\mathrm{i}^{*}\right\}$ and $\mathrm{i}^{*}$ is the fault or the class of fault that has been identified. Note that it is possible to distinguish theoretically and $2 \mathrm{p}(\mathrm{K}+1)$ fault over a different time $\mathrm{K}$, in practice this number will be much smaller given the length of propagation. So here arises the problem of choosing the number of state variables to undergo this process of detection / identification, such as the problem of choosing the sampling period to ensure a relative speed of propagation of error sufficient (sampling must be fast enough to help identify and monitor the failure of one moment to another).

If $\mathrm{D}^{*} \neq 0, \mathrm{I}(0)^{*}$ defines the set of plausible faults of order 0 .

We can also introduce sets of plausible faults order of $d$ assuming the duration of the outage has been misjudged:

$$
\min _{i \in I \mid \tau_{i}=\tau} \sum_{h=1}^{\tau+1+d} \sum_{j=1}^{p}\left(m_{j h}^{k}-p_{j h}\right)^{2}=\Delta(\mathrm{d})^{*} \text { obtained } \mathrm{i} \in \mathrm{I}(\mathrm{d})^{*}
$$

So :

$$
\min _{d>0} \Delta(d)^{*}<D^{*} \quad \text { obtained } \mathrm{d}^{*}
$$

It is probable that the duration of propagation of the fault current has been underestimated $\mathrm{d}^{*}$ units:

$$
\hat{\tau}=\tau+d^{*}
$$

\section{APPLICATION TO ROTOCRAFT FAULT DETECTION}

The considered system is shown in Figure 3 where rotors one and three are clockwise while rotors two and four are counter clockwise. The main simplifying assumptions adopted with respect to flight dynamics in this study are a rigid cross structure, constant wind, negligible aerodynamic contributions resulting from translational speed, no ground effect as well as small air density effects and negligible response times for the rotors. It is then possible to write the rotorcraft flight equations as follows.

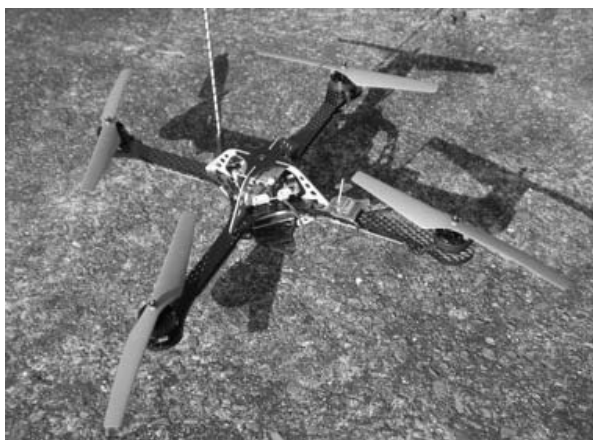

Figure 3. The considered rotorcraft

Here we have applied the state distribution generation method proposed in section 4 . It has been supposed that the nine components of the state of the discrete version rotorcraft are measured while the first component of this state is the flat output from which the other two state components can be reconstructed (here $\mathrm{p}=1$ ) for one period before current decision time. To generate an initial distribution using the flatness relations and take into account the errors present in the flat outputs measurements, two values have been chosen 
for each output randomly to activate relation (30), leading to different initial estimates.

Figure 4 displays the corresponding error histograms, showing that the Gaussian hypothesis for modeling errors in equation (25) is acceptable in the current case. Finally this leads us to adopt the following values for the different modeling errors which are assumed to be statistically independent:

$$
\begin{aligned}
& W_{1}=0.8255 \quad W_{4}=1.0989 W_{7}=1.6364 \\
& W_{2}=0.9963 \quad W_{5}=1.3109 W_{8}=0.8719 \\
& W_{3}=0.7809, W_{6}=0.944 \quad W_{9}=0.8381
\end{aligned}
$$

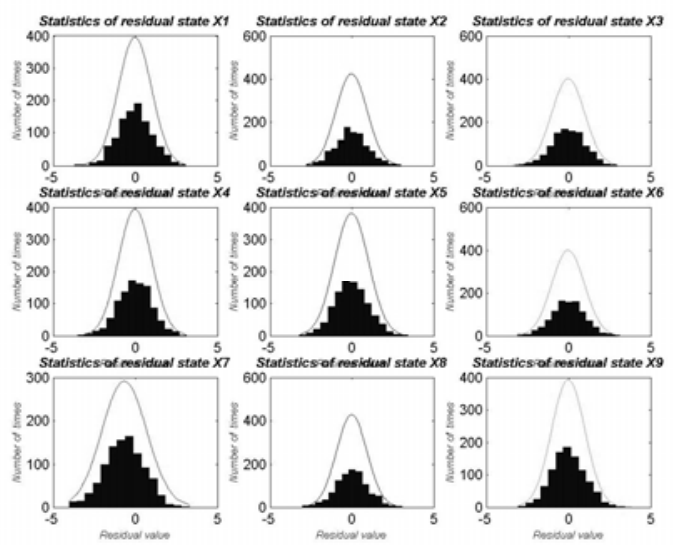

Figure 4. State error histograms

\section{The Case Study Considered}

Here we apply the state distribution generation method proposed. It is supposed that the nine components of the state of the discrete version rotorcraft are measured while the first component of this state is the flat output from which the other two state components can be reconstructed (here $p=1$ ) for one period before current decision time. To generate an initial distribution using the flatness relations and take into account the errors present in the flat outputs measurements, 2 values have been chosen for each output randomly to activate relation (30), leading to: $2^{m \cdot(p+q+1)}=2^{8}=256$ different initial estimates. Then applying twice relation (33) we get at current time a state distribution of $256 \times 2=512$ samples. These 512 samples are generated on line at each discrete instant and allow to estimate probabilistic distributions so that a fault test such as (44) can be performed by comparison with the direct measurements of $x_{4}(=p), x_{5}(=q)$ and $x_{6}(=r)$.

Figure 5 displays tests relative to $x_{5}$ at one times when no fault has happened. In that case the generated distributions for measurements and estimation of state $x_{5}$ adopt very close central values.

Figure 6 displays the same tests when at time 0 a faulty event induces a $10 \%$ loss of mass for the rotorcraft with consequences on the inertia parameters $I_{x x}$ and $I_{y y}$. The different graphics show that after the fault, the probability to detect it increases with time since the discrepancy between the pairs of generated distributions widen. Then a decision logic based on a reduced number of results from successive tests can be established so that false detection occurrence is diminished while the fault detection delay remains small.

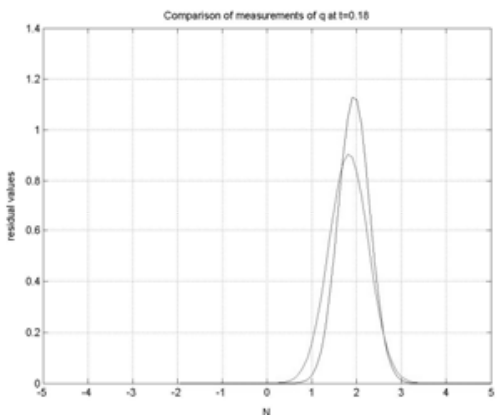

Figure 5. Distribution comparison without fault

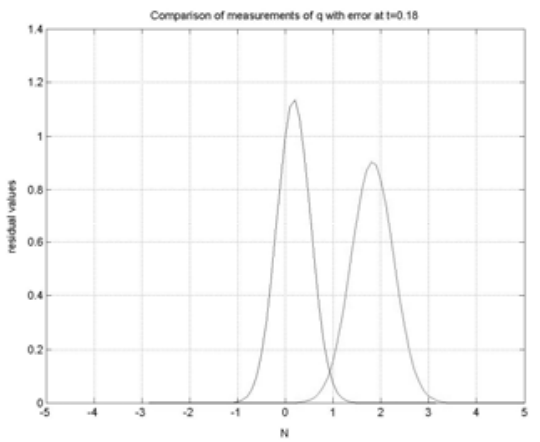

Figure 6. Distribution comparison with fault

\section{CONCLUSION}

This communication proposes a new approach to detect faults occurring in nonlinear systems whose discrete dynamics is difference flat. The proposed approach can be improved in different ways:

Other distribution generation schemes could be considered easily and compared with the one adopted here.

The generated distribution could be used directly in the fault detection tests avoiding the Gaussian hypothesis which has been adopted here for sake of simplicity. Then, the effect over the state uncertainty of the nonlinearities present in a difference flat system could be taken fully into account.

This approach can be extended to fault localization diagnostic by taking into account estimations and measurements performed at successive periods of time.

With respect to applications in the field of flight systems, it has been already shown that part of general aircraft flight dynamics as well as Quadri-Rotor flight dynamics can be approximated by difference flat models. In fact this was the 
main motivation to develop the above fault detection method and the proposed approach has been applied to rotorcraft fault detection.

\section{REFERENCES}

[1] [1] Hoffmann, G., Rajnarayan, D.G., Waslander, S. L., Dostal , D., Jang, J.S. and Tomlin, C. J., The Standford Tetsbed of Autonomous Rotorcraft for Multi-Agent Control, 23rd Digital Avionics Systems Conference, Salt Lake City, UT, November 2004.

[2] [2] M. Fliess, J. Lévine, P. Martin, P. Rouchon, Flatness and defect of non-linear systems: introductory theory and examples, INT. J. CONTROL, 1995, Vol.61, No. 6, 1327-1361.

[3] [3] Hassan K. Khalil, Nonlinear Systems, Prentice Hall, 3rd Ed., 2002.

[4] [4] W. Lu, F. Mora-Camino, M. de Coligny, K. Achaibou, Flight Mechanics and Differential Flatness, DINCON 2004, Ilha Solteira, Brasil, pp. 830-839, 2004.

[5] [5] Hoffmann, G., Rajnarayan, D.G., Waslander, S. L., Dostal , D., Jang, J.S. and Tomlin, C. J., The Standford Tetsbed of Autonomous Rotorcraft for Multi-Agent Control, 23rd Digital Avionics Systems Conference, Salt Lake City, UT, November 2004

[6] [6] Fliess M., Lévine J., Martin Ph., Rouchon P., Sur les systèmes non linéaires différentiellement plats", C.R. Acad. Sci. Paris, 315, série I : 619-624., 1992.

[7] [7] Clark R.N., Instrument fault detection, IEEE Trans., Aerospace and Electronic System, EAS-14, pp. 558-563, 1975

[8] [8] Frank P.M., Fault diagnosis in dynamic systems via state estimation- a survey, In Tzafestas S., Singh M., Schmidt G. (Eds.) Systems fault diagnostics, reliability and related knowledge-based approaches, 1, pp. 35-98, 1987.

[9] [9] W. Lu, F. Mora-Camino, K. Achaibou, Differential Flatness and Flight Guidance: A Neural Adaptive Approach, AIAA, Guidance Navigation and Control Conference, San Francisco, 15-18 août 2005

[10] [10] N. Zhang, A. Drouin, A. Doncescu and F. Mora-Camino, Differential flatness approach for Rotorcraft Fault Detection, Chinese Control Conference, July 2008, Kunming, China.

[11] [11] N. Zhang and F. Mora-Camino, Rotorcraft Fault Detection Using Difference Flatness, AIAA Guidance Navigation and Control, Conference, 2009 Chicago, American.

[12] [12] N. Zhang and F. Mora-Camino, Fault Detection Using Difference Flatness, DINCON'09, 8th Brazilian Conference on Dynamics Control and Applications, May 18-22, 2009.

[13] [13] Staroswiecki M., Cocquempot V., Cassa J.P. Observer based and parity space approaches for failure detection and identification. Proc. Of the IMACS/IFAC Symposium on Modelling and Control of Technological Systems, Lille, France, vol. 25, pp. 536-541, 1991. 